\title{
Weathering a Macrophage Storm
}
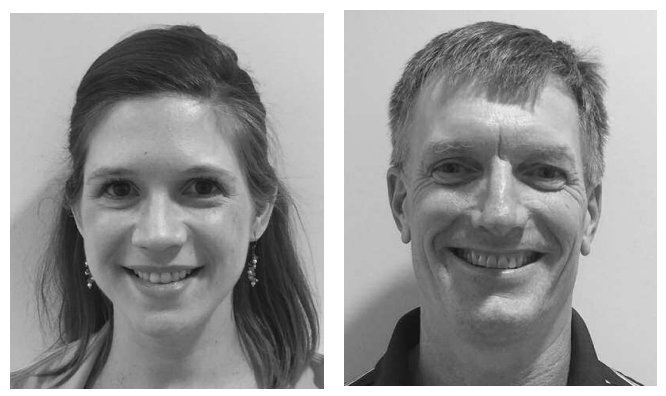

In rheumatology circles, macrophage activation syndrome (MAS) describes a potentially life-threatening complication of systemic inflammatory disorders, frequently making matters worse for those with systemic juvenile idiopathic arthritis (sJIA) and its adult counterpart, adult-onset Still disease (AOSD). A dysfunctional immune response results in continuous activation and expansion of T lymphocytes and macrophages, leading to an overproduction of proinflammatory cytokines (a cytokine storm) and resulting in multiorgan dysfunction. Like the related disorder, secondary hemophagocytic lymphohistiocytosis (HLH), MAS features include high, unremitting fever, hyperferritinemia, hepatosplenomegaly, lymphadenopathy, pancytopenia, and hypofibrinogenemia. Other laboratory abnormalities include elevated liver enzymes, D-dimers, lactate dehydrogenase, and triglycerides (TGC). Soluble interleukin 2 receptor $\alpha$ (sCD25) may be elevated, but testing is often not available at an on-site laboratory and therefore is not routinely done at the time of diagnosis s $^{1,2,3,4}$. Histopathology often reveals characteristic increased hemophagocytic activity in the bone marrow (and other tissues), with positive CD163 (histiocyte) staining, although this feature is often not present in initial stages and is neither highly sensitive nor specific for MAS ${ }^{5,6}$.

Most commonly studied as a complication of sJIA, the reported prevalence of MAS is estimated to be about $10 \%$; however, reports suggest subclinical MAS may be present in $30 \%-40 \%$ of patients with sJIA $^{7,8}$. There are several diagnostic challenges in the early recognition of MAS, particularly in distinguishing it from a flare of sJIA or AOSD. Moreover, there is no single pathognomonic feature of MAS, and many clinical features and laboratory abnormalities overlap with those of systemic inflammatory disorders, making it difficult to distinguish the underlying disease from the life-threatening comorbidity ${ }^{1,2,3,4}$. Further, until very recently, there were no universal validated criteria to aid in diagnosis. However, patient data-based classification criteria for MAS in patients with sJIA were recently reported ${ }^{1,9}$. The criteria were developed in an iterative process over several years and relied on real patient data and controls ( $>1100$ subjects from 33 countries). The final criteria were chosen by MAS experts selecting from nearly 1000 computer-generated combinations of clinical and laboratory findings. The final MAS criteria for children with sJIA proved to be both sensitive (0.73) and specific (0.99). The diagnosis of MAS can be made in a febrile patient with sJIA or suspected sJIA who has a serum ferritin level $>684 \mathrm{ng} / \mathrm{ml}$ plus any 2 of the following: platelet count $\leq 181 \times 10^{9} / \mathrm{l}$, aspartate aminotransferase $>48$ units/l, TGC $>156 \mathrm{mg} / \mathrm{dl}$, or fibrinogen $\leq 360 \mathrm{mg} / \mathrm{dl}$ (Table 1$)^{1,9}$. These relatively few total criteria are routinely readily available and timely.

The clinical overlap of MAS and secondary HLH has led some practitioners to use the longer-standing HLH-2004 diagnostic guidelines, which require 5 of the following 8 criteria to be met for diagnosis: fever, splenomegaly, cytopenias (affecting $\geq 2$ of 3: hemoglobin $<90 \mathrm{~g} / \mathrm{l}$, platelets $<100 \times 10^{9} / 1$, neutrophils $\left.<1.0 \times 10^{9} / 1\right)$, hypertriglyceridemia $(\geq 265 \mathrm{mg} / \mathrm{dl})$ and/or hypofibrinogenemia $(\leq 1.5 \mathrm{~g} / \mathrm{l})$, hemophagocytosis in bone marrow or spleen or lymph nodes, low or absent natural killer cell activity, ferritin $\geq 500 \mu \mathrm{g} / \mathrm{l}$, and $\mathrm{sCD} 25 \geq 2400 \mathrm{units} / \mathrm{ml}^{10,11}$. Using this strict set of criteria may delay diagnosis in patients with a less severe initial presentation, putting them at risk for rapid clinical decline and potential death from delayed treatment. To improve upon this, Fardet, et al developed a modified scoring system (HScore) ${ }^{12}$, based on the HLH-2004 criteria, to estimate a patient's risk of having secondary HLH; however, this has not been validated in patients with an underlying rheumatic systemic inflammatory condition such as sJIA or AOSD. In fact, a majority of patients included in this study had HLH secondary to an underlying malignancy or infection ${ }^{12}$. Nevertheless, the HScore is being explored in other clinical settings of children and adults with secondary $\mathrm{HLH}^{13,14}$.

In this issue of The Journal, Ahn, et al ${ }^{15}$ present an

See MAS in AOSD, page 996

Personal non-commercial use only. The Journal of Rheumatology Copyright @ 2017 . All rights reserved. 
Table 1. Classification criteria for macrophage activation syndrome in known or suspected systemic juvenile idiopathic arthritis. From Ravelli, et al. Ann Rheum Dis 2016;75:481-9; and Ravelli, et al. Arthritis Rheumatol 2016;68:566-76; adapted with permission ${ }^{1,9}$.

Classification Criteria for MAS

- $\quad$ Fever AND ferritin $>684 \mathrm{ng} / \mathrm{ml}$

Plus any 2 of the following 4 criteria:

- $\quad$ Platelet count $\leq 181 \times 10^{9} / 1$

- $\quad$ AST $>48$ units $/ 1$

- Triglycerides $>156 \mathrm{mg} / \mathrm{dl}$

- $\quad$ Fibrinogen $\leq 360 \mathrm{mg} / \mathrm{dl}$

MAS: macrophage activation syndrome; AST: aspartate aminotransferase

expansion of the 2016 European League Against Rheumatism/American College of Rheumatology/Pediatric Rheumatology International Trials Organization (EULAR/ACR/ PRINTO) classification criteria for MAS in patients with sJIA to include the related disorder of AOSD. They retrospectively analyzed 64 hospitalized patients with AOSD and fever and classified them as having MAS based on the 2016 sJIA MAS criteria ${ }^{1,9,15}$. They further divided the patients into 2 categories: those with MAS prior to admission, and those who developed MAS after admission. Ahn, et al also calculated the HScore to estimate a patient's risk of having MAS/secondary HLH and compared it to those with MAS and those without MAS based on the 2016 pediatric sJIA MAS criteria. They found that $56.2 \%$ of hospitalized patients with AOSD met criteria for sJIA associated MAS. Of the 36 patients with MAS, $61.1 \%$ had MAS at the time of admission, and the remaining $38.8 \%$ developed MAS during the hospitalization ${ }^{15}$. Clinical manifestations were similar between the groups; however, the erythrocyte sedimentation rate and platelet counts were lower, and lactate dehydrogenase and TGC levels were higher, in patients with MAS at the time of admission, similar to what others have seen in $\mathrm{AOSD}^{16}$.

Survival rates were lower in AOSD patients with MAS (66.6\%) compared to those without MAS (100\%). Mortality was higher in patients with MAS at the time of admission, with a majority of these resulting from multiorgan failure as opposed to secondary infection. A higher-fold increase in ferritin, defined as the maximum ferritin value divided by baseline ferritin level, was also associated with lower survival rates. Similarly, AOSD MAS patients with an Hscore $\geq 80 \%$ had a higher mortality rate than those with scores $<80 \%$. Treatment was more aggressive in AOSD patients with MAS compared to those without, and included higher glucocorticoid dosage and more frequent use of cyclosporine, etoposide, and intravenous immunoglobulin ${ }^{15}$.

Only 26 (18 with MAS and 8 without MAS) of the 64 AOSD patients had a bone marrow biopsy performed. Hemophagocytosis was found in 9 of the 18 AOSD patients with MAS (50\%) and in 0 patients without MAS ${ }^{15}$. These findings are consistent with literature suggesting that bone marrow hemophagocytosis is not highly sensitive in confirming MAS $(\sim 60 \%)$ and may delay diagnosis and ultimately, treatment ${ }^{5,6}$. Additionally, strict application of the HLH-2004 criteria classified only 7 patients as having MAS, meaning MAS would have gone unrecognized in 29 patients. Thus, the HLH-2004 criteria are likely insensitive for diagnosing MAS in the setting of AOSD, and at a minimum, may cause a delay in treatment, thereby potentially increasing mortality rates ${ }^{15}$.

The study by Ahn, et al explores the clinical crossover of sJIA and AOSD and attempts to expand the 2016 classification criteria for MAS, which have been validated only in pediatric patients with $\mathrm{sJIA}^{1}$, to include adult patients with AOSD $^{15}$. Based on these results, the 2016 EULAR/ACR/ PRINTO MAS criteria may be clinically useful in patients with AOSD. These criteria may thus aid in a prompt diagnosis and subsequent aggressive and targeted treatment of MAS to better improve longterm morbidity and mortality in those with AOSD. The 2016 MAS criteria are based largely on laboratory values, with fever being the only clinical manifestation ${ }^{1}$. Some of these laboratory abnormalities can be seen in other systemic inflammatory conditions, such as systemic lupus erythematosus (SLE) and Kawasaki disease, making it difficult to generalize the criteria to include all rheumatologic conditions. It is therefore important to have additional validation studies to determine the clinical relevance of these criteria with respect to other specific inflammatory conditions.

Validated MAS criteria for rheumatic conditions will allow for more controlled treatment studies. To date, there is no standardized treatment protocol, and management remains largely anecdotal. Traditionally, high-dose corticosteroids have been the mainstay of treatment. Addition of cyclosporine has also been used and may be beneficial in patients refractory to steroids $s^{1,2,3,4}$. Etoposide has been included as standard treatment per HLH-1994 and HLH-2004 protocols; however, there are significant risks associated with cytotoxic chemotherapy, and it may not be the best choice for MAS patients with underlying rheumatologic disorders ${ }^{10,11}$. By comparison, several recent case series have suggested targeted interleukin 1 inhibition with anakinra may be a safe and effective first-line therapy for controlling MAS ${ }^{17,18,19}$. To better study safety and efficacy of treatments, it is crucial to validate classification criteria for various inflammatory disorders to be used in clinical trials.

With a mortality rate of $8 \%$ among patients with sJIA, and likely a notably higher one for those with AOSD, it is important for practitioners to recognize MAS early and to treat it aggressively in hopes of improving patient survival ${ }^{20}$. While this current AOSD study has several limitations, including a small patient sample, restriction to hospitalized patients, and lack of treatment outcomes, it serves as an initial study to explore the clinical relevance of the 2016 sJIA MAS

Personal non-commercial use only. The Journal of Rheumatology Copyright (C) 2017. All rights reserved. 
criteria in patients with AOSD, a related systemic inflammatory condition ${ }^{15}$. With additional studies, it may be possible to apply modified MAS criteria for sJIA patients to those with pediatric and adult SLE, Kawasaki disease, spondyloarthritis, etc., with the potential for earlier recognition and treatment, in hopes of reducing overall mortality for patients with rheumatic disorders.

\section{COURTNEY B. CRAYNE, MD; RANDY Q. CRON, MD, PhD, \\ Division of Rheumatology, Department of Pediatrics, University of Alabama at Birmingham, Birmingham, Alabama, USA.}

Address correspondence to Dr. R.Q. Cron, Children's of Alabama, Division of Rheumatology, 1600 7th Ave. S., CPP \#M10, Birmingham, Alabama 35233-1711, USA; E-mail: rcron@peds.uab.edu

\section{REFERENCES}

1. Ravelli A, Minoia F, Davì S, Horne A, Bovis F, Pistorio A, et al. 2016 classification criteria for macrophage activation syndrome complicating systemic juvenile idiopathic arthritis: a European League Against Rheumatism/American College of Rheumatology/Paediatric Rheumatology International Trials Organisation collaborative initiative. Ann Rheum Dis 2016; 75:481-9.

2. Ravelli A, Davì S, Minoia F, Martini A, Cron RQ. Macrophage activation syndrome. Hematol Oncol Clin North Am 2015; 29:927-41.

3. Davì S, Minoia F, Pistorio A, Horne A, Consolaro A, Rosina S, et al. Performance of current guidelines for diagnosis of macrophage activation syndrome complicating systemic juvenile idiopathic arthritis. Arthritis Rheumatol 2014;66:2871-80.

4. Ravelli A, Grom AA, Behrens EM, Cron RQ. Macrophage activation syndrome as part of systemic juvenile idiopathic arthritis: diagnosis, genetics, pathophysiology and treatment. Genes Immun 2012;13:289-98

5. Goel S, Polski JM, Imran H. Sensitivity and specificity of bone marrow hemophagocytosis in hemophagocytic lymphohistiocytosis. Ann Clin Lab Sci 2012;42:21-5.

6. Ho C, Yao X, Tian L, Li FY, Podoltsev N, Xu ML. Marrow assessment for hemophagocytic lymphohistiocytosis demonstrates poor correlation with disease probability. Am J Clin Pathol 2014;141:62-71.

7. Behrens EM, Beukelman T, Paessler M, Cron RQ. Occult macrophage activation syndrome in patients with systemic juvenile idiopathic arthritis. J Rheumatol 2007;34:1133-8.

8. Bleesing J, Prada A, Siegel DM, Villanueva J, Olson J, Ilowite NT, et al. The diagnostic significance of soluble CD163 and soluble interleukin-2 receptor alpha-chain in macrophage activation syndrome and untreated new-onset systemic juvenile idiopathic arthritis. Arthritis Rheum 2007;56:965-71.
9. Ravelli A, Minoia F, Davì S, Horne A, Bovis F, Pistorio A, et al. 2016 classification criteria for macrophage activation syndrome complicating systemic juvenile idiopathic arthritis: a European League Against Rheumatism/American College of Rheumatology/Paediatric Rheumatology International Trials Organisation Collaborative Initiative. Arthritis Rheumatol 2016;68:566-76.

10. Henter JI, Horne A, Arico M, Egeler RM, Filipovich AH, Imashuku S, et al. HLH-2004: diagnostic and therapeutic guidelines for hemophagocytic lymphohistiocytosis. Pediatr Blood Cancer 2007;48:124-31.

11. Rosado FG, Kim AS. Hemophagocytic lymphohistiocytosis: an update on diagnosis and pathogenesis. Am J Clin Pathol 2013;139:713-27.

12. Fardet L, Galicier L, Lambotte O, Marzac C, Aumont C, Chahwan $\mathrm{D}$, et al. Development and validation of the HScore, a score for the diagnosis of reactive hemophagocytic syndrome. Arthritis Rheumatol 2014;66:2613-20.

13. Debaugnies F, Mahadeb B, Ferster A, Meuleman N, Rozen L, Demulder A, et al. Performances of the H-Score for diagnosis of hemophagocytic lymphohistiocytosis in adult and pediatric patients. Am J Clin Pathol 2016;145:862-70.

14. Cattaneo C, Oberti M, Skert C, Passi A, Farina M, Re A, et al. Adult onset hemophagocytic lymphohistiocytosis prognosis is affected by underlying disease and coexisting viral infection: analysis of a single institution series of 35 patients. Hematol Oncol 2016 Jun 3 (E-pub ahead of print).

15. Ahn SS, Yoo BW, Jung SM, Lee SW, Park YB, Song J. Application of the 2016 EULAR/ACR/PRINTO Classification Criteria for Macrophage Activation Syndrome in patients with adult-onset Still disease. J Rheumatol 2017;44:996-1003.

16. Bae CB, Jung JY, Kim HA, Suh CH. Reactive hemophagocytic syndrome in adult-onset Still disease: clinical features, predictive factors, and prognosis in 21 patients. Medicine 2015;94:e451.

17. Miettunen PM, Narendran A, Jayanthan A, Behrens EM, Cron RQ. Successful treatment of severe paediatric rheumatic disease-associated macrophage activation syndrome with interleukin-1 inhibition following conventional immunosuppressive therapy: case series with 12 patients. Rheumatology 2011;50:417-9.

18. Bruck N, Suttorp M, Kabus M, Heubner G, Gahr M, Pessler F. Rapid and sustained remission of systemic juvenile idiopathic arthritis-associated macrophage activation syndrome through treatment with anakinra and corticosteroids. J Clin Rheumatol 2011;17:23-7.

19. Kelly A, Ramanan AV. A case of macrophage activation syndrome successfully treated with anakinra. Nat Clin Pract Rheumatol 2008;4:615-20.

20. Minoia F, Davi S, Horne A, Demirkaya E, Bovis F, Li C, et al. Clinical features, treatment and outcome of macrophage activation syndrome complicating systemic juvenile idiopathic arthritis: a multinational, multicenter study of 362 patients. Arthritis Rheumatol 2014;66:3160-9.

J Rheumatol 2017;44:970-2; doi:10.3899/jrheum.170370 